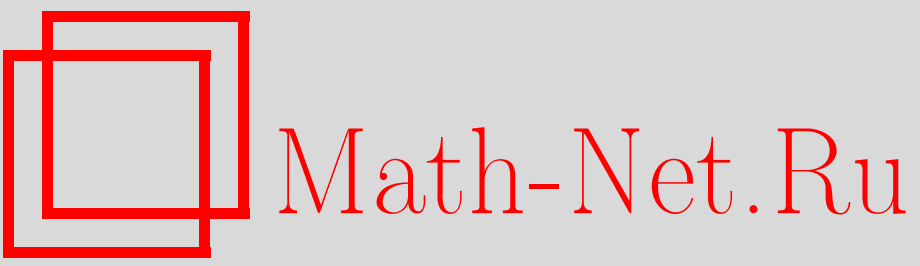

Б. Г. Конопельченко, Л. Мартинес Алонсо, Е. Медина, Квазиконформные отображения и решения бездисперсионной иерархии КП, ТМФ, 2002, том 133, номер 2, 247-258

DOI: https://doi.org/10.4213/tmf394

Использование Общероссийского математического портала Math-Net.Ru подразумевает, что вы прочитали и согласны с пользовательским соглашением

http://www. mathnet.ru/rus/agreement

Параметры загрузки :

IP: 54.80 .73 .141

26 апреля 2023 г., 14:04:41 


\section{КВАЗИКОНФОРМНЫЕ ОТОБРАЖЕНИЯ И РЕШЕНИЯ БЕЗДИСПЕРСИОННОЙ ИЕРАРХИИ КП}

$\bar{\partial}$-Формализм, используемый для исследования бездисперсионных интегрируемых иерархий применен к бездисперсионной иерархии Кадомцева-Петвиашвили (КП). Этот формализм привязывается к теории квазиконформных отображений на плоскости, что позволяет описать некоторые классы явных решений бездисперсионной иерархии КП.

Ключевые слова: бездисперсионные иерархии, квазиконформные отображения, $\bar{\partial}$-уравнения.

\section{1. ВВЕДЕНИЕ}

Бездисперсионные, или квазиклассические, интегрируемые иерархии занимают существенное место в теории интегрируемых систем. Они лежат в основе многочисленных подходов к решению задач, возникающих в физике и математике (см., например, [1]-[8]). Эти иерархии связаны с некоторыми классическими задачами теории конформных отображений [9]. Для исследования бездисперсионных интегрируемых иерархий недавно был предложен $\bar{\partial}$-метод [10], [11]. Этот метод позволяет обнаружить глубокую взаимосвязь этих иерархий и теории квазиконформных отображений [12]-[15].

Наш подход основан на нелинейном $\bar{\partial}$-уравнении

$$
S_{\bar{z}}=W\left(z, \bar{z}, S_{z}\right)
$$

где $z \in \mathbb{C}$, а $S(z, \bar{z}, \mathbf{t})$ представляет собой комплекснозначную функцию, зависящую от бесконечного набора $\mathbf{t}$ параметров (времен), $S_{\bar{z}}:=\partial S / \partial \bar{z}, S_{z}:=\partial S / \partial z, W$ - подходящая функция переменных $z, \bar{z}$ и $S_{z}$.

Уравнение (1) предполагает, что первые производные от функции $S$ по параметрам $\mathbf{t}$ удовлетворяют семейству уравнений Бельтрами

$$
f_{\bar{z}}=\mu(z, \mathbf{t}) f_{z}
$$

* Dipartimento di Fisica, Universita di Lecce and Sezione INFN, Lecce, Italy

${ }^{\dagger}$ Departamento de Física Teórica II, Universidad Complutense, Madrid, Spain

‡Departamento de Matemáticas, Universidad de Cádiz, Puerto Real, Cádiz, Spain 
где

$$
\mu:=W^{\prime}\left(z, \bar{z}, S_{z}\right), \quad W^{\prime}=W_{\xi}(z, \bar{z}, \xi) .
$$

Это обеспечивает связь между $\bar{\partial}$-методом и теорией квазиконформных отображений, которая основана на уравнении Бельтрами. Заметим, что уравнение (1) также хорошо известно из теории квазиконформных отображений (см., например, [16]).

Метод данной работы основан на нахождении решений уравнения (1) с использованием классических методов решения уравнений с частными производными первого порядка типа Гамильтона-Якоби. В данной работе в рамках этого метода будут явно найдены некоторые частные решения бездисперсионной иерархии Кадомцева-Петвиашвили (КП). Также будет показано, что даже простейшее из этих решений (см. пример 1 из раздела 4) не может быть получено с помощью стандартных методов, основанных на технике преобразования годографа [2].

\section{2. БЕЗДИСПЕРСИОННЫЕ ИЕРАРХИИ КП}

Бездисперсионная иерархия КП представляет собой следуюшую классическую версию пары уравнений Лакса для стандартной теории КП [1]-[7]:

$$
\frac{\partial z}{\partial t_{n}}=\left\{\Omega_{n}, z\right\}, \quad \Omega_{n}(p, \mathbf{t}):=\left(z^{n}\right)_{+}, \quad n \geqslant 1 .
$$

Здесь $z=z(p, \mathbf{t})$ представляет собой комплекснозначную функцию от комплексного переменного $p$ и от бесконечного набора $\mathbf{t}:=\left(t_{1}, t_{2}, \ldots\right)$ комплексных параметров, которая допускает разложение в ряд Лорана вида

$$
z=p+\sum_{n \geqslant 1} \frac{a_{n}(\mathbf{t})}{p^{n}}, \quad p \rightarrow \infty,
$$

а через $\left(z^{n}\right)_{+}$обозначена полиномиальная часть разложения $z^{n}$ в ряд по $p$,

$$
(z)_{+}=p, \quad\left(z^{2}\right)_{+}=p^{2}+2 a_{1}, \quad\left(z^{3}\right)_{+}=p^{3}+3 p a_{1}+3 a_{2} \quad \text { и т.д. }
$$

Скобка Пуассона определена как

$$
\{F, G\}:=\frac{\partial F}{\partial p} \frac{\partial G}{\partial x}-\frac{\partial F}{\partial x} \frac{\partial G}{\partial p}, \quad x:=t_{1} .
$$

Условия совместности системы (4) имеют вид

$$
\frac{\partial \Omega_{m}}{\partial t_{n}}-\frac{\partial \Omega_{n}}{\partial t_{m}}+\left\{\Omega_{m}, \Omega_{n}\right\}=0, \quad m \neq n .
$$

Рассмотрим два интересных примера нелинейных уравнений бездисперсионной иерархии КП. 
1. В случае $n=2$ уравнение (4) сводится к импульсным уравнениям Бенни

$$
\frac{\partial a_{n+1}}{\partial t}+\frac{\partial a_{n+2}}{\partial x}+n a_{n} \frac{\partial a_{1}}{\partial x}=0, \quad t:=-2 t_{2}
$$

2. Условия совместности (6) в случае, когда $n=2$ и $m=3$, приводят собственно к бездисперсионному уравнению КП (уравнению Заболоцкой-Хохлова)

$$
\left(u_{t}-\frac{3}{2} u u_{x}\right)_{x}=\frac{3}{4} u_{y y}, \quad u:=2 a_{1}, \quad t:=t_{3}, \quad y:=t_{2} .
$$

Из условий (6) следует (см. [6]), что для всякого решения $z=z(p, \mathbf{t})$ бездисперсионной иерархии КП найдется соответствующая функция $S=S(z, \mathbf{t})$ такая, что

$$
\frac{\partial S(z, \mathbf{t})}{\partial t_{n}}=\Omega_{n}(p(z, \mathbf{t}), \mathbf{t}), \quad n \geqslant 1
$$

где $p=p(z, \mathbf{t})$ получается, если обратить решение $z=z(p, \mathbf{t})$. Без потери обшности будем предполагать, что функция $S$ допускает разложение Лорана вида

$$
S(z, \mathbf{t})=\sum_{n \geqslant 1} z^{n} t_{n}+\sum_{n \geqslant 1} \frac{S_{n}(\mathbf{t})}{z^{n}}, \quad z \in \Gamma,
$$

где $\Gamma=\{z:|z|=r\}$. Заметим, что полагая $n=1$ в (9) и используя разложение (10), можно получить, что $p=p(z, \mathbf{t})$ записывается в виде

$$
p=\frac{\partial S(z, \mathbf{t})}{\partial x}=z+\sum_{n \geqslant 1} \frac{b_{n}(\mathbf{t})}{z^{n}}, \quad b_{n}:=\frac{\partial S_{n}}{\partial x} .
$$

Обратно, если задана функция $S=S(z, \mathbf{t})$, которая удовлетворяет условиям (9) и (10), то можно доказать [4], что функция $z=z(p, \mathbf{t})$, обратная по отношению к функции $p=$ $p(z, \mathbf{t})$ в (11), задает решение бездисперсионной иерархии КП.

Здесь и далее будем называть функции, удовлетворяющие условиям (9) и (10), $S$ функи иями бездисперсионной иерархии КП. Они связаны с тау-функциями [4], посколькy

$$
S(z, \mathbf{t})=\sum_{n \geqslant 1} z^{n} t_{n}+\sum_{n \geqslant 1} \frac{1}{n z^{n}} \frac{\partial \ln \tau(\mathbf{t})}{\partial t_{n}}, \quad z \in \Gamma .
$$

Заметим, что система уравнений (9) для $S$-функции бездисперсионной иерархии КП представляет собой набор согласованных уравнений типа Гамильтона-Якоби

$$
\frac{\partial S}{\partial t_{n}}=\Omega_{n}\left(\frac{\partial S}{\partial x}, \mathbf{t}\right), \quad n \geqslant 2,
$$

которые описывают квазиклассический предел линейной системы уравнений на волновую функцию стандартной иерархии КП. 
В работах [2], [4], [6] были предложены различные способы построения решений бездисперсионной иерархии КП с помощью $S$-функций. В данной работе используется $\bar{\partial}$-метод, предложенньй в работах [10], [11], в рамках которого $S$-функции описываются с помошью решений $\bar{\partial}$-уравнений (1). Напомним, что симметрии уравнения (1) (вариации первого порядка $f:=\delta S$ ) описываются в этом подходе с помощью семейства уравнений Бельтрами (2), (3). Из этого свойства следует, в частности, что все первые производные $\partial S / \partial t_{n}$ решения уравнения (1) удовлетворяют уравнениям (2).

Рассмотрим локальное решение $f$ уравнения Бельтрами. Если $f$ имеет ненулевой якобиан в некоторой точке $z_{0}$, то все гладкие локальные решения $F$ в окрестности $z_{0}$ имеют вид аналитических функций от $f: F=F(f)$. Из этого свойства следует, что при подходяших условиях те решения $S \bar{\partial}$-уравнения (1), которые допускают разложения вида (10), также удовлетворяют условиям (12). Таким образом, эти решения задают $S$-функции бездисперсионной иерархии КП. Ниже данная схема описана более строго.

\section{3. КВАЗИКОНФОРМНЫЕ ОТОБРАЖЕНИЯ}

Квазиконформные отображения представляют собой естественное и весьма богатое расширение конщепции конформных отображений. Напомним некоторые базисные свойства квазиконформных отображений (см., например, [10]-[15]).

Пусть $\mu=\mu(z)$ - измеримая функция на области $G$ комплексной плоскости такая, что почти всюду на $G$ справедливо неравенство $|\mu(z)|<k$ для некоторого числа $k, 0<$ $k<1$. Функция $f=f(z)$ называется квазиконформныцм отображением с комплексныц растяжением $\mu$ в области $G$, если выполняются следуюшие условия:

1) $f$ представляет собой гомеоморфизм $f: D \rightarrow D^{\prime}$;

2) $f$ есть обобшенное решение линейного уравнения Бельтрами (2) на $D$, причем частные производные $f_{\bar{z}}$ и $f_{z}$ представляют собой функции, локально интегрируемые с квадратом.

Свойства решений уравнения Бельтрами (2) хорошо известны (см., например, [13]). Некоторые из этих свойств принципиально важны для нашего рассмотрения. Но прежде чем описать эти свойства, введем оператор Кальдерона-Зигмунда [13]

$$
(T h)(z):=\frac{1}{2 \pi i} \iint_{\mathbb{C}} \frac{h\left(z^{\prime}\right)}{\left(z^{\prime}-z\right)^{2}} d z^{\prime} \wedge d \bar{z}^{\prime},
$$

где интеграл берется по Коши в смысле главного значения. Тогда имеет место следуюший фундаментальньй результат [13].

Tеорема 1. Для любого $p \geqslant 2$ оператор $T$ задает ограниченный оператор в пространстве $L^{p}(\mathbb{C})$. Более того, норма $\|T\|_{p}$ непрерывна по отношению $к$ параметру р и удовлетворяет условию

$$
\lim _{p \rightarrow 2}\|T\|_{p}=1 .
$$

Эта теорема утверждает, что для любого $0 \leqslant k<1$ сушествует $\delta(k)>0$ такое, что $k\|T\|_{p}<1$ для всех $2<p<2+\delta(k)$.

Следуюшая теорема формулирует свойство квазиконформных отображений, которое оказывается важным для описания $\bar{\partial}$-метода. 
Теорема 2. Пусть $\mu$-измеримая функиия с компактныцм носителем, содержащимся внутри круга $|z|<R$, и такая, что $\|\mu\|_{\infty}<k<1$. Тогда для любого $p>2$ такого, что $k\|T\|_{p}<1$, единственным обобщенным решением уравнения Бельтрами (2), для которого справедливь условия

$$
f(z)=O\left(\frac{1}{z}\right), \quad z \rightarrow \infty
$$

$u f_{\bar{z}}, f_{z} \in L^{p}(\mathbb{C})$, бyдem $f \equiv 0$.

Этот результат представляет собой утверждение о единственности из формулировки теоремы о сушествовании так называемого нормального решения уравнения Бельтрами [12]-[14]. Доказательство этого утверждения основано на том, что оператор $T$ задает действие $\partial_{z} \partial_{\bar{z}}^{-1}$ на пространстве $L^{p}(\mathbb{C})$, и по предположению теоремы получим, что уравнение Бельтрами для $f$ преврашается в интегральное уравнение

$$
\phi-\mu T \phi=0, \quad \phi:=f_{\bar{z}},
$$

на $L^{p}(\mathbb{C})$. Тогда поскольку $\|\mu T\|_{p} \leqslant k\|T\|_{p}<1$, то получим, что $\phi \equiv 0$, и с учетом свойства (15) имеем $f \equiv 0$.

Вернемся теперь к $\bar{\partial}$-уравнению (1), предполагая, что $W\left(z, \bar{z}, S_{z}\right)=0$ для всех $z$, лежаших вне круга $\Gamma=\{z:|z|=r\}$. Допустим, что известно решение $S=S(z, \bar{z}, \mathbf{t})$ уравнения (1) внутри диска $D=\{z:|z|<r\}$ с граничным условием $\left.S\right|_{\Gamma}:=S\left(z, r^{2} / z, \mathbf{t}\right)$ вида (10) и такое, что множество

$$
\Omega:=\left\{\mathbf{t}: \sup _{z \in D} \mid W^{\prime}\left(z, \bar{z}, S_{z}(z, \mathbf{t}) \mid \leqslant k\right\}\right.
$$

непусто при некотором $k, 0<k<1$. В этом случае можно применить теорему 2 к уравнению Бельтрами (2), (3). Более того, принимая во внимание, что

$$
\frac{\partial S}{\partial t_{n}}=z^{n}+\sum_{m \geqslant 1} \frac{\partial S_{m}(\mathbf{t})}{\partial t_{n}} z^{-m}, \quad z \in \Gamma
$$

получим, что эти функции допускают непрерывное продолжение из $D$ в аналитические функции вне контура $Г$. Тем самым эти функции задают решения системы (2), (3) во всей плоскости комплексного переменного. С другой стороны, ясно, что

$$
\frac{\partial S}{\partial t_{n}}-\left(z^{n}\right)_{+}=\frac{\partial S}{\partial t_{n}}-\Omega_{n}\left(\frac{\partial S}{\partial x}, \mathbf{t}\right)=O\left(\frac{1}{z}\right), \quad z \rightarrow \infty,
$$

апотому из теоремы 2 следует, что выполняется условие (9), откуда получаем, что функция $S=S(z, \bar{z}, \mathbf{t})$ задает $S$-функцию бездисперсионной иерархии КП для $\mathbf{t} \in \Omega$. 


\section{4. РЕШЕНИЯ БЕЗДИСПЕРСИОННОЙ ИЕРАРХИИ КП}

Чтобы построить явные решения бездисперсионной иерархии КП, рассмотрим $\bar{\partial}$-уравнения вида

$$
S_{\bar{z}}=\theta(r-|z|) V\left(z, \bar{z}, S_{z}\right)
$$

где $r>0, \theta(\xi)$ - стандартная функция Хевисайда, а $V$ - аналитическая функция от $z, \bar{z}$ и $S_{z}$. Решение строится следуюшим образом.

1. Найдем сначала решения $S=S(z, \mathbf{a})$ уравнения (16),

$$
S_{\bar{z}}=V\left(z, \bar{z}, S_{z}\right), \quad|z|<r,
$$

зависящие от набора свободных параметров $\mathbf{a}:=\left(a_{0}, a_{1}, \ldots\right)$.

2. Затем выберем те решения, граничные значения которых на круге $\Gamma=\{z:|z|=r\}$ имеют вид (10).

Уравнение (17) представляет собой уравнение с частными производными типа Гамильтона-Якоби, и мы можем использовать хорошо развитые методы построения решений таких уравнений. Например, если $V=V\left(S_{z}\right)$ содержит зависимость только от $S_{z}$, то из уравнения (17) следует, что

$$
m_{\bar{z}}=V_{m}(m) m_{z}, \quad m:=S_{z} .
$$

Это уравнение легко решается, если применить метод характеристик. Общее решение уравнения (17) при этом неявно параметризуется:

$$
\begin{gathered}
S=V(m) \bar{z}+m z-f(m), \\
V_{m} \bar{z}+z=f_{m}(m),
\end{gathered}
$$

где $f=f(m)$ - произвольная функция. Заметим, что из второго уравнения системы (18) следует, что

$$
f_{m}\left(m_{0}\right)=z, \quad m_{0}:=\left.m(z, \bar{z})\right|_{\bar{z}=0},
$$

и тогда $f_{m}\left(m_{0}\right)$ представляет собой функцию, обратную $m_{0}=m_{0}(z)$.

Чтобы построить явные решения, надо еше больше сузить класс возможных решений. Например, исследуем случай, когда в рассмотрении участвует только лишш конечный набор из $N+1$ параметров $\mathbf{a}=\left(a_{0}, a_{1}, \ldots, a_{N}\right)$. Тогда возникает проблема вычленить те решения $S$, в которых в формуле (10) не появляется членов $z^{n} t_{n}$ при $n>N$. В других случаях решения $\bar{\partial}$-уравнения могут зависеть от времен $t_{n}, n>N$, которые сами по себе являются функциями от $t_{1}, \ldots, t_{N}$, но на этом пути невозможно получить решение бездисперсионной иерархии КП.

Начнем исследование возможных допустимых случаев с рассмотрения подкласса $\bar{\partial}$-уравнений (17) вида

$$
S_{\bar{z}}=\bar{z}^{s} \sum_{m \geqslant 0}^{M} p_{m}(z)\left(S_{z}\right)^{m}, \quad|z|<r,
$$


где $s \geqslant 0, M \geqslant 2$, коэффициенты $p_{m}=p_{m}(z)$ суть многочлены от переменной $z$, причем $p_{M} \not \equiv 0$. Будем искать решение уравнения (19) в виде следуюшего ряда:

$$
S=\sum_{n \geqslant 0} c_{n}(z) \bar{z}^{n(s+1)}
$$

где $c_{0}$ - произвольный многочлен $N$-й степени $(N \geqslant 2)$,

$$
c_{0}(z)=\sum_{n=0}^{N} a_{n} z^{n}, \quad a_{N} \neq 0
$$

Подставляя (20) в (19), получим рекуррентное соотношение

$$
c_{n+1}=\frac{1}{(n+1)(s+1)} \sum_{m \geqslant 0}^{M} p_{m}(z)\left(\sum_{r_{1}+\cdots+r_{m}=n} c_{r_{1}}^{\prime} \ldots c_{r_{m}}^{\prime}\right), \quad n \geqslant 0
$$

из которого следует, что все коэффициенты $c_{n}(z)$ в выражении (20) имеют вид многочленов. Чтобы найти вид функции $S$ на границе $|z|=r$, необходимо определить степени этих многочленов.

ЛЕмма. Если степени коэффициентов $p_{m}$ в (19) удовлетворяют условиям

$$
\operatorname{deg} p_{m} \leqslant(M-m)(N-1), \quad m=0,1, \ldots, M
$$

mo

$$
\operatorname{deg} c_{n}=n[M(N-1)-N]+N, \quad n \geqslant 0 .
$$

ДокАЗАТЕЛЬСтво. Применим метод математической индукции. Очевидно, что условие (23) выполнено при $n=0$. Предположим, что это условие выполняется при $n^{\prime} \leqslant n$, и рассмотрим члены в выражении $(22)$ для $c_{n+1}$. Принимая во внимание, что $r_{1}+\cdots+r_{m}=n$, получим

$$
\begin{aligned}
\operatorname{deg}\left(p_{m} c_{r_{1}}^{\prime} \ldots c_{r_{m}}^{\prime}\right) & =\sum_{i=1}^{m}\left[r_{i}[M(N-1)-N]+N-1\right]+\operatorname{deg} p_{m}= \\
& =n[M(N-1)-N]+m(N-1)+\operatorname{deg} p_{m} \leqslant \\
& \leqslant n[M(N-1)-N]+M(N-1)=(n+1)[M(N-1)-N]+N
\end{aligned}
$$

Более того, так как $p_{M}$ есть ненулевая константа, ясно, что соответствуюшие члены в формуле (22) удовлетворяют условию

$$
\operatorname{deg}\left(p_{M} c_{r_{1}}^{\prime} \ldots c_{r_{M}}^{\prime}\right)=n[M(N-1)-N]+M(N-1)=(n+1)[M(N-1)-N]+N
$$

Тем самым можно заключить, что условие (23) выполнено и для коэффициента $c_{n+1}$, что завершает доказательство. 
Предполагая, что ряд (20) сходится при некотором $r \geqslant 0$, найдем, что в предположениях леммы непрерывное продолжение функции $S$ на границу $|z|=r$ имеет вид

$$
S=\sum_{n \geqslant 0} r^{2 n(s+1)} \frac{c_{n}(z)}{z^{n(s+1)}}, \quad \frac{c_{n}(z)}{z^{n(s+1)}}=O\left(z^{d_{n}}\right),
$$

где

$$
d_{n}=n[M(N-1)-N-s-1]+N
$$

Поскольку решение, отвечаюшее (21), зависит от $N+1$ свободных параметров $\left(a_{0}, \ldots, a_{N}\right)$, интерес представляют лишь те случаи, в которых $d_{n} \leqslant N$ при всех $n \geqslant 0$. Из формулы (25) очевидно, что это возможно, только если

$$
N \leqslant \frac{M+s+1}{M-1}
$$

Например, полагая $s=0$, получим, что возможны только три случая, приведенные в таблице.

\begin{tabular}{|c|c|c|}
\hline$(M, N)$ & $V\left(z, S_{z}\right)$ & $\left.S\right|_{\bar{z}=0}$ \\
\hline$(2,2)$ & $\alpha\left(S_{z}\right)^{2}+\left(\sum_{i=0}^{1} \beta_{i} z^{i}\right) S_{z}+\sum_{i=0}^{2} \gamma_{i} z^{i}$ & $\sum_{i=0}^{2} a_{i} z^{i}$ \\
\hline$(3,2)$ & $\alpha\left(S_{z}\right)^{3}+\left(\sum_{i=0}^{1} \beta_{i} z^{i}\right)\left(S_{z}\right)^{2}+\left(\sum_{i=0}^{2} \gamma_{i} z^{i}\right) S_{z}+\sum_{i=0}^{3} \eta_{i} z^{i}$ & $\sum_{i=0}^{2} a_{i} z^{i}$ \\
\hline$(2,3)$ & $\alpha\left(S_{z}\right)^{2}+\left(\sum_{i=0}^{2} \beta_{i} z^{i}\right) S_{z}+\sum_{i=0}^{4} \gamma_{i} z^{i}$ & $\sum_{i=0}^{3} a_{i} z^{i}$ \\
\hline
\end{tabular}

ПримеР 1. Простейший случай из класса решений $(2,2)$ отвечает уравнению

$$
S_{\bar{z}}=\theta(1-|z|)\left(S_{z}\right)^{2}
$$

где $\left.S\right|_{\bar{z}=0}$ - многочлен второго порядка. Этот случай дает

$$
S= \begin{cases}\frac{1}{2} \frac{(z-b)^{2}}{a-2 \bar{z}}-c, & |z| \leqslant 1 \\ \frac{1}{2} \frac{z(z-b)^{2}}{a z-2}-c, & |z| \geqslant 1 .\end{cases}
$$

Из условия регулярности функции $S$ внутри единичного круга следует, что $|a|>2$.

На границе $|z|=1$ получим

$$
S=\frac{1}{2 a} z^{2}+\left(\frac{1}{a^{2}}-\frac{b}{a}\right) z+\frac{2}{a^{3}}+\frac{b^{2}}{2 a}-\frac{2 b}{a^{2}}-c+O\left(\frac{1}{z}\right) .
$$

Таким образом, из условия, что ответ должен иметь необходимый вид $S$-функции бездисперсионной иерархии КП, следуют отождествления

$$
x=\frac{1}{a^{2}}-\frac{b}{a}, \quad t_{2}=\frac{1}{2 a}, \quad c=\frac{2}{a^{3}}+\frac{b^{2}}{2 a}-\frac{2 b}{a^{2}} .
$$


С другой стороны, масштабное преобразование с комплексным параметром соответствующего уравнения Бельтрами (1), (2) имеет вид

$$
\mu(z, \bar{z}):=2 \theta(1-|z|) \frac{z-b}{a-2 \bar{z}} .
$$

С учетом вышесказанного получим следующее ограничение:

$$
|\mu(z, \bar{z})|<2 \frac{|b|+1}{|a|-2}, \quad z \in \mathbb{C} .
$$

Таким образом, для любого $0<k<1$ имеем $|\mu(z)| \leqslant k$, если только $k|a|>2(|b|+k+1)$. Тем самым доказано, что в пространстве параметров сушествует не пустая область, в которой уравнение Бельтрами (1), (2) удовлетворяет условиям, обсуждавшимся в разделе 3 . Это означает, что

$$
p:=S_{x}=\frac{z^{2}-4 t_{2} z+2\left(x+4 t_{2}^{2}\right)}{z-4 t_{2}}, \quad|z|=1,
$$

и мы получаем следуюшее решение для $t_{2}$-потока (потока Бенни) бездисперсионной иерархии КП:

$$
z=\frac{p}{2}+2 t_{2}+\sqrt{\left(\frac{p}{2}-2 t_{2}\right)^{2}-2 x-8 t_{2}^{2}} .
$$

Заметим, что зависимость от переменных времен входит в это решение через две функции $u_{1}=2 t_{2}$ и $u_{2}=-2 x-8 t_{2}^{2}$, поскольку это решение переписывается в виде

$$
z=\frac{p}{2}+u_{1}+\sqrt{\left(\frac{p}{2}-u_{1}\right)^{2}+u_{2}} .
$$

Если бы это решение могло быть получено с помощью метода годографа [2], то оно бы соответствовало такой редукции бездисперсионной иерархии КП, в которой функции $\mathbf{u}=\left(u_{1}, u_{2}\right)$ удовлетворяли бы диагонализуемой системе уравнений гидродинамического типа с инвариантами Римана, задаваемыми нулями функции $\partial z(p, \mathbf{u}) / \partial p$. Но эта функция не имеет нулей при $u_{2} \neq 0$, поскольку

$$
\frac{\partial z(p, \mathbf{u})}{\partial p}=\frac{p / 2-u_{1}+\sqrt{\left(p / 2-u_{1}\right)^{2}+u_{2}}}{2 \sqrt{\left(p / 2-u_{1}\right)^{2}+u_{2}}} .
$$

Тем самым можно заключить, что решение (29) бездисперсионной иерархии КП не может быть получено в рамках метода годографа. 
Пример 2. $\bar{\partial}-У$ равнение

$$
S_{\bar{z}}=\theta(1-|z|)\left(S_{z}\right)^{3},
$$

где $\left.S\right|_{\bar{z}=0}$ - многочлен второго порядка, представляет собой пример случая $(3,2)$. Соответствуюшая $S$-функция имеет вид

$$
S=\bar{z} m^{3}+(z-b) m-\frac{a}{2} m^{2}-c, \quad|z|<1,
$$

где

$$
m=\frac{1}{6 \bar{z}}\left(a-\sqrt{a^{2}-12(z-b) \bar{z}}\right) .
$$

Соотношения, определяюшие параметры $x, t_{2}$ бездисперсионной иерархии КП, имеют вид

$$
x=\frac{b}{6}\left(\sqrt{a^{2}-12}-a\right), \quad t_{2}=\frac{1}{108}\left(18 a-a^{3}+\left(a^{2}-12\right)^{3 / 2}\right) .
$$

Соответствуюшее решение $t_{2}$-потока бездисперсионной иерархии КП есть

$$
\begin{aligned}
z= & a\left(a-\sqrt{a^{2}-12}\right) \frac{p}{12}+\frac{3 x}{\sqrt{a^{2}-12}}+\frac{1}{12}\left[\left(a\left(a-\sqrt{a^{2}-12}\right) p+\frac{36 x}{\sqrt{a^{2}-12}}\right)^{2}-\right. \\
& \left.-12\left(\left(a-\sqrt{a^{2}-12}\right) p+\frac{3\left(a+\sqrt{a^{2}-12}\right) x}{\sqrt{a^{2}-12}}\right)^{2}\right]^{1 / 2},
\end{aligned}
$$

где $a=a\left(t_{2}\right)$ получается из (31).

ПримеР 3 . Класс решений $(2,3)$ представляет наибольший интерес, поскольку он содержит решения бездисперсионной иерархии КП, зависяшие от параметров $x, t_{2}, t_{3}$. Рассмотрим уравнение

$$
S_{\bar{z}}=\theta(1-|z|)\left(S_{z}\right)^{2},
$$

в котором $\left.S\right|_{\bar{z}=0}$ есть многочлен третьего порядка. Выбирая $m_{0}:=\left.S_{z}\right|_{\bar{z}}=a z^{2}+b z+c$ и используя параметризацию (18), получим

$$
f(m)=-\frac{b}{2 a} m+\frac{1}{12 a^{2}}\left(4 a m+b^{2}-4 a c\right)^{3 / 2}+d=-\frac{b}{2 a} m+\frac{1}{12 a^{2}}(4 a \bar{z} m+2 a z+b)^{3}+d
$$

$$
m=\frac{1}{8 \bar{z}^{2}}\left[\frac{1}{a}-4\left(z+\frac{b}{2 a}\right) \bar{z}-\sqrt{\frac{4}{a}\left(\frac{b^{2}}{a}-4 c\right) \bar{z}^{2}-\frac{8}{a}\left(z+\frac{b}{2 a}\right) \bar{z}+\frac{1}{a^{2}}}\right] .
$$

Тем самым получим, что

$$
S=\left(z+\frac{b}{2 a}\right) m+\bar{z} m^{2}-\frac{1}{12 a^{2}}(4 a \bar{z} m+2 a z+b)^{3}-d, \quad|z|<1 .
$$

Теперь становится ясно, что для того чтобы функция $S$ была непрерывной, необходимо потребовать [11], чтобы

$$
\frac{4}{a}\left(\frac{b^{2}}{a}-4 c\right) \bar{z}^{2}-\frac{8}{a}\left(z+\frac{b}{2 a}\right) \bar{z}+\frac{1}{a^{2}} \neq 0, \quad|z|<1 .
$$


Заметим также, что функция $S$ регулярна в начале координат, так как $\lim _{z \rightarrow 0} m=c$.

Приведем теперь схему вычисления $u=-2 \partial S_{1} / \partial x$. Сначала надо вычислить несколько первых членов в разложении функции $S$ при $|z|=1$. Для этого полезно использовать следующее тождество:

$$
S_{z}=m-\left(\frac{m}{z}\right)^{2}, \quad|z|=1
$$

Теперь, раскладывая $m$ при $|z|=1$ и полагая

$$
S_{z}=3 t_{3} z^{2}+2 t_{2} z+x-\frac{S_{1}}{z^{2}}+\cdots, \quad|z|=1
$$

в формуле (36), получим

$$
\begin{gathered}
3 t_{3}=-\frac{3}{4}+\frac{3}{8 a}+\frac{1}{32 a^{2}}\left((1-8 a)^{3 / 2}-1\right), \quad 2 t_{2}=\frac{b}{8 a^{2}}(1-4 a-\sqrt{1-8 a}), \\
x=-\frac{b^{2}}{8 a^{2}}\left(1+\frac{4 a-1}{2 \sqrt{1-8 a}}\right)+\frac{c}{4 a}(1-\sqrt{1-8 a})
\end{gathered}
$$

И

$$
S_{1}=\frac{\left(2 b^{2}+(1-8 a) c\right)^{2}}{(1-8 a)^{5 / 2}}
$$

Из этих выражений следует, что $u=-2 \partial S_{1} / \partial x$ дается выражением $\left(t:=t_{3}, y:=t_{2}\right)$

$$
u=\frac{4(5-12 t+4 \sqrt{1-12 t})^{2}\left((-1+12 t) x-4 y^{2}\right)}{3(1+4 t)(1-12 t+2 \sqrt{1-12 t})^{3}}
$$

которое удовлетворяет системе (8). Заметим, что в соответствии с работой [8] решение (38) принадлежит классу решений бездисперсионного уравнения КП, которые задают структуры Эйнштейна-Вейля, конформные по отношению к метрике Эйнштейна.

Более общее рассмотрение решений, допускающих разложение (19), но теперь уже с $s \neq 0$ и с нетривиальной зависимостью от переменной $z$ в правой части, будет проведено в последующих работах.

Благодарности. Б. К. благодарен организаторампрограммы "Интегрируемые системы" за финансовую поддержку. Работа Б. К. была частично поддержана грантом COFIN 2000 “Sintesi”. Пребывание Л. М. А. в Университете г. Кембриджа было профинансировано Fundación Banco Bilbao Vizcaya Argentaria. Работа Е. М. была частично поддержана CICYT (проект PB98-0821). 


\section{Список литературы}

[1] В. Е. Захаров. Функц. анализ и его прилож. 1980. Т. 14. № 2. С. 15-24; Physica D. 1981. V. 3. P. 193-202; P.D. Lax, C. D. Levermore. Commun. Pure Appl. Math. 1983. V. 36. P. 253-290; P. 571-593; P. 809-830.

[2] Y. Kodama. Phys. Lett. A. 1988. V. 129. P. 223-226; 1990. V. 147. P. 477-482; И. М. Крuчевер. Функц. анализ и его прилож. 1988. Т. 22. № 3. С. 37-52; I. M. Krichever. Commun. Pure Appl. Math. 1994. V. 47. P. 437-475.

[3] Б. А. Дубровин, С. П. Новиков. УМН. 1989. Т. 44. № 6. С. $29-98$.

[4] K. Takasaki, T. Takebe. Int. J. Mod. Phys. A. 1992. V. 1. Suppl. 1B. P. 889-922; Rev. Math. Phys. 1995. V. 7. P. 743-818.

[5] N. M. Ercolani et al. (eds.). Singular Limits of Dispersive Waves. Nato ASI Ser. B. V. 320. New York: Plenum, 1994.

[6] I. M. Krichever. Commun. Math. Phys. 1992. V. 143. P. 415-429.

[7] B. A. Dubrovin. Commun. Math. Phys. 1992. V. 145. P. 195-203; B. A. Dubrovin, Y. Zhang. Commun. Math. Phys. 1998. V. 198. P. 311-361; Normal forms of hierarchies of integrable PDEs, Frobenious manifolds and Gromov-Witten invariants. math.DG/0108160.

[8] M. Dunaiski, L. J. Mason, P. Tod. J. Geom. Phys. 2001. V. 37. P. 63-93.

[9] J. Gibbons, S. P. Tsarev. Phys. Lett. A. 1999. V. 258. P. 263-271; P. B. Wiegmann, A. Zabrodin. Commun. Math. Phys. 2000. V. 213. P. 523-538.

[10] B. Konopelchenko, L. Martinez Alonso, O. Ragnisco. J. Phys. A. 2001. V. 34. P. 10209-10217.

[11] B. Konopelchenko, L. Martinez Alonso. Phys. Lett. A. 2001. V. 286. P. 161-166.

[12] И. Н. Векуа. Обобщенные аналитические функции. 2-е изд. М.: Наука, 1988.

[13] L. V. Ahlfors. Lectures on Quasi-Conformal Mappings. Princeton, NJ: Van Nostrand, 1966.

[14] O. Lehto. Univalent Functions and Teichmüller Spaces. Berlin: Springer, 1987.

[15] Q. Lehto, K. I. Virtanen. Quasiconformal Mappings in the Plane. Berlin: Springer, 1973.

[16] B. Bojarski. Symp. Math. 1976. V. 18. P. 458-499; T. Iwaniec. Symp. Math. 1976. V. 18. P. 501-517. 